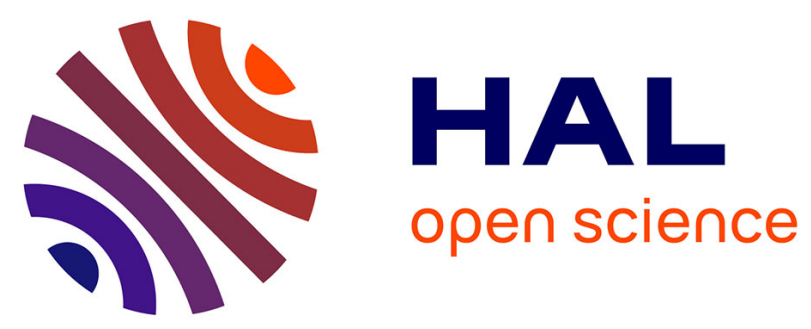

\title{
CHAMPS DE PRESSION DES TRANSDUCTEURS PLANS DANS LE DOMAINE DE L'ACOUSTIQUE NON-LINÉAIRE : THÉORIE ET EXPÉRIENCE
}

S. Nachef, D. Cathignol, J. Chapelon

\section{- To cite this version:}

S. Nachef, D. Cathignol, J. Chapelon. CHAMPS DE PRESSION DES TRANSDUCTEURS PLANS DANS LE DOMAINE DE L'ACOUSTIQUE NON-LINÉAIRE : THÉORIE ET EXPÉRIENCE. Journal de Physique IV Proceedings, 1992, 02 (C1), pp.C1-645-C1-648. 10.1051/jp4:19921139 . jpa00251096

\section{HAL Id: jpa-00251096 https://hal.science/jpa-00251096}

Submitted on 1 Jan 1992

HAL is a multi-disciplinary open access archive for the deposit and dissemination of scientific research documents, whether they are published or not. The documents may come from teaching and research institutions in France or abroad, or from public or private research centers.
L'archive ouverte pluridisciplinaire HAL, est destinée au dépôt et à la diffusion de documents scientifiques de niveau recherche, publiés ou non, émanant des établissements d'enseignement et de recherche français ou étrangers, des laboratoires publics ou privés. 


\title{
CHAMPS DE PRESSION DES TRANSDUCTEURS PLANS DANS LE DOMAINE DE L'ACOUSTIQUE NON-LINÉAIRE : THÉORIE ET EXPÉRIENCE
}

\author{
S. NACHEF ${ }^{*, * *}$, D. CATHIGNOL ${ }^{* *}$ et J.Y. CHAPELON** \\ "TECHNOMED INTERNATIONAL, Parc d'Activités du Chêne, Bd. des Droits de l'Homme, \\ F-69500 Bron, France \\ ${ }^{* *}$ INSERM Unité 281, 151 Cours Albert Thomas, F-69424 Lyon cedex 03, France
}

\begin{abstract}
The aim of this paper is the study of the validity of the parabolic KZK equation in the case of finite amplitude ultrasound pressure field generated by piezoelectric axisymmetric plane source at different excitation levels. The numerical implementation used to solve the parabolic KZK equation is based on a finite difference method which accounts for diffraction, absorption and nonlinear effects. Comparisons between measurements and computed results are made along and across the acoustic axis for the first three harmonics of the wave forms. Good agreement is shown for low pressure levels, but the disagreement becomes more and more important according to the excitation level and consequently to the increase of the pressure at the source. Finally, the evolution of the amplitude of the first three harmonics is exhibited along the acoustic axis. It is shown that their minima and maxima move toward the source when we increase the excitation level.
\end{abstract}

\begin{abstract}
Résumé Le but de cet article est l'étude de la validité de l'approximation parabolique de l'équation KZK dans le cas du champ de pression à amplitude finie généré par un transducteur piézo-électrique plan à symétrie axial, à différents niveaux d'excitation. La mise en application numérique pour résoudre ce problème est basée sur une méthode de différences finies tenant compte des phénomènes de diffraction, d'absorption et de nonlinéarité. Des comparaisons entre l'expérience et les résultats calculés sont effectuées le long de l'axe acoustique et perpendiculairement à celui-ci, pour les trois premiers harmoniques du profil temporel de l'onde déformée. Un bon accord est observé pour les faibles niveaux de pression, mais le désaccord devient de plus en plus important lorsqu'on augmente la tension d'excitation et donc la pression générée à la source. Enfin, nous traçons des réseaux de courbes expérimentales montrant l'évolution de l'amplitude de ces harmoniques en fonction du niveau d'excitation, le long de l'axe acoustique. Nous observons que leurs minima et maxima se décalent vers la source lorsque le niveau d'excitation augmente.
\end{abstract}

\section{I - INTRODUCTION}

Le calcul du champ de pression généré par une source ultrasonore excitée à hauts niveaux de tension doit tenir compte des effets non-linéaires ainsi que de la diffraction et de l'absorption dans le milieu de propagation. A faible niveau de pression, le problème peut être traité par la théorie quasi-linéaire qui suppose uniquement la génération du second harmonique. Il est clair qu'à moyen et haut niveaux de pression, comme ceux observés dans le domaine de la thérapie (hyperthermie, ablathermie, lithotritie ...) ou de l'acoustique sous-marine (sonar, ...), la théorie basee sur l'approche d'une perturbation simple n'est plus valable et il devient nécessaire de tenir compte des interactions produites entre les harmoniques générés par le phénomène non-linéaire.

L'outil utilisé pour le calcul du champ de pression est la résolution numérique de l'approximation parabolique de l'équation KZK. Le but de ce papier est d'étudier les limites de validité de cette approximation à travers les comparaisons des résultats calculés et les mesures effectuées pour une très grande gamme de niveau d'excitation.

Nous comparons les résultats théoriques et expérimentaux le long et perpendiculairement à l'axe acoustique pour les trois premiers harmoniques et nous traçons des réseaux de courbes expérimentales montrant l'évolution de l'amplitude de ces harmoniques en fonction du niveau d'excitation. 


\section{II - THEORIE}

L'approximation parabolique de l'équation d'onde non-linéaire tenant compte des effets de diffraction, d'absorption et de non linéarité a été proposée par Zabolotskaya et Khokhlov [1]. Kuznetsov [2] a introduit l'absorption dans la même équation qui est considérée actuellement comme l'une des équations de base de l'acoustique non-linéaire. Pour étudier la génération d'harmoniques dans le champ ultrasonore, l'équation KZK a été reprise par Naze Tjøtta et Tjøtta [3] et transformée en un système de coordonnées non dimensionnelles pour permettre sa résolution numérique. Aanonsen et al. [3] a été le premier à résoudre cette équation par une méthode de différences finies pour le champ proche d'une source circulaire supposée vibrer sinusoïdalement et en piston. Cependant, cette approximation a des limites théoriques, elle exige que le rayon de la source soit très grand devant la longueur d'onde et demeure inefficace très près de la source et très loin de l'axe acoustique. Hamilton et al. [4] a introduit une transformation de coordonnées pour étendre la méthode au champ lointain. Hart et al. [5] a examiné le cas du champ convergent en introduisant des transformations convergente dans la zone pré-focale et divergente dans la zone postfocale. Baker et al. [6] a comparé les résultats numériques avec l'expérience et a obtenu un bon accord à faible niveau de pression pour une source plane. Plus tard, les champs pulsés ont été étudiés par Baker et al. [7] et Neighbors et al. [8], et plus récemment, nous avons étudié [9] les limites de validité de l'approximation parabolique en comparant les résultats numériques et expérimentaux dans le cas d'une source peu focalisée excitée en monochromatique à des niveaux d'excitation de plus en plus élevés. Ce travail a pour but d'étudier les limites de cette approximation pour une source plane excitée en monochromatique lorsqu'on augmente le niveau d'excitation.

\section{III - DISPOSITIF EXPERIMENTAL}

L'expérimentation est effectuée dans une cuve d'eau de $90 \mathrm{~cm}$ de long, $90 \mathrm{~cm}$ de large et $40 \mathrm{~cm}$ de profondeur. L'eau étant déionisée et dégazée.

La source ultrasonore est un disque piézo-électrique plan de $50 \mathrm{~mm}$ de diamètre et de $1 \mathrm{MHz}$ de fréquence principale de résonance.

La mesure de pression est effectuée à l'aide d'un hydrophone en PVDF de type bilaminaire dont la partie active est de $0,5 \mathrm{~mm}$ de diamètre, fabriqué par la société Marconi Research - UK.

Des moteurs pas à pas de $10 \mu \mathrm{m}$ de résolution sont commandés pour piloter un système de micropositionnement qui permet de déplacer l'hydrophone dans le champ ultrasonore le long des trois axes $x, y$ et $\mathrm{z}$. La direction $\mathrm{z}$ positive est prise comme direction de propagation.

Un générateur de signaux (HP 8116A) est utilisé pour exciter le transducteur avec une trentaine de sinusoïdes à $1 \mathrm{MHz}$ répétées toutes les $100 \mathrm{~ms}$. Cette solution permet de considérer que nous sommes en régime permanent sans risque de détériorer l'hydrophone. Un amplificateur de puissance $5 \mathrm{~kW}$ (Ampar 5002 , société Prâna R\&D - F) permet d'atteindre la pression de 20 Bars environ à la surface de la source.

La sortie de l'hydrophone est reliée directement à l'entrée d'un oscilloscope (Tektronix - 2430A DSO) qui est programmé pour enregistrer une partie du signal acoustique située dans le régime permanent. Ensuite, ce signal est transféré dans un ordinateur où sa densité spectrale est calculée par transformation de Fourier. Ainsi, on obtient la contribution de la fréquence considérée au profil de l'onde au point de mesure. On peut également mesurer la phase absolue de chaque harmonique en prenant comme référence de phase le même signal sinusoïdal pour tous les points de mesure.

Tout le système est commandé par un ordinateur compatible IBM PC - AT à travers des interfaces IEEE permettant d'effectuer des cycles automatiques de Positionnement / Enregistrement / Transfert.

\section{IV - RESULTATS ET DISCUSSIONS}

Dans un premier temps, les résultats de la résolution numérique ont été comparés à très faible pression aux résultats numériques d'une autre théorie d'acoustique linéaire axée sur l'étude de la diffraction d'une source circulaire plane [10]. Les courbes simulées des deux méthodes se superposent parfaitement, aussi bien sur l'axe acoustique que perpendiculairement à celui-ci (non données ici).

Dans un deuxième temps, nous avons étudié la validité de l'approximation parabolique dans le cas des faibles non-linéarités en affrontant les résultats théoriques et expérimentaux. Nous avons constaté que la théorie tient parfaitement compte des phénomènes physiques en jeux et donne une estimation très précise de la pression mesurée dans le champ acoustique où l'approximation parabolique est valable [6] et [9].

Nous avons également effectué des comparaisons à des niveaux de pression supérieurs à ceux utilisés par Baker [6] pour voir de quelle manière la théorie commence à diverger des résultats expérimentaux. 
Au vu des résultats obtenus (Figures 1, 2 et 3), nous constatons que le modèle tient parfaitement compte du phénomène de diffraction puisqu'il se rend compte des moindres détails des variations des amplitudes de la fréquence principale et de ses premiers harmoniques. De plus, bien que l'amplitude de la fréquence principale soit bien estimée (Figure 1), les niveaux de pression calculés des deux premiers harmoniques sous-estiment la mesure (Figures 2 et 3 ). Plus la pression à la source augmente, plus la divergence entre la théorie et l'expérience devient importante.

Pour observer l'évolution de l'amplitude de la fréquence principale et de ses premiers harmoniques le long et perpendiculairement à l'axe acoustique, nous avons tracé des réseaux de courbes expérimentales de ces grandeurs en fonction de la tension d'excitation. Nous avons observé une augmentation considérable de l'amplitude des harmoniques dans le champ très proche; tout se passe comme s'il existait un phénomène de sur-focalisation. De plus, nous avons observé un décalage vers la source des minima et des maxima des harmoniques sur l'axe acoustique. Notons que ce décalage est d'autant plus grand que l'ordre de l'harmonique est élevé (Figures 4,5 et 6).

l'approximation parabolique n'est pas applicable à ces niveaux de pression. Néanmoins, elle peut toujours donner une idée de la forme générale du champ acoustique sans fournir des informations précises sur les niveaux de pression.

Les résultats expérimentaux des trois dernières figures peuvent avoir deux interprétations. La première interprétation repose sur le phénomène de l'augmentation de la célérité des ultrasons dans le milieu de propagation lorsque celui-ci est soumis à des pressions ou des contraintes très élevées, cette augmentation de célérité peut puiser ses origines dans le phénomène de streaming (déplacement de la matière) qui augmente avec la pression et affecte d'une manière plus importante les harmoniques d'ordres plus élevés. Cependant, bien que cette interprétation puisse expliquer le décalage des minima et des maxima mentionné plus haut, elle ne peut expliquer l'augmentation de l'amplitude des harmoniques dans le champ très proche. La deuxième interprétation repose sur l'hypothèse de l'augmentation du coefficient de non linéarité qui expliquerait l'augmentation de l'amplitude des harmoniques mais pas le décalage des minima.

\section{$\mathrm{V} \cdot$ - CONCLUSION}

Nous pouvons conclure que l'approximation parabolique de l'équation KZK tient parfaitement compte du phénomène de diffraction, même à fortes pressions, mais qu'elle s'écarte des résultats expérimentaux quant à l'estimation du phénomène non-linéaire. Bien qu'elle donne une idée générale du champ de pression, elle ne peut estimer avec précision la pression dès lors que cette pression est élevée. Pour améliorer ce modèle, il y a la possibilité de pousser l'approximation du terme non-linéaire au troisième ordre ou d'introduire un terme supplémentaire qui tiendra compte du phénomène de streaming.

\section{REFERENCES}

1. E. A. Zabolotskaya and R. V. Khokhlov, "Quasi-plane waves in the nonlinear acoustics of confined beams", Sov. Phys. Acouct. 15, 35-40 (1969).

2. V. P. Kuznetsov, "Equations of nonlinear acoustics", Sov. Phys. Acouct. 16, 467-470 (1970).

3. S. I. Aanonsen, T. Barkve, J. Naze Tjøtta and S. Tjøtta, "Distribution and harmonic generation in the nearfield of a finite amplitude sound beam", J. Acouct. Soc. Am. 75 749-768 (1984).

4. M. F. Hamilton, J. Naze Tjøtta and S. Tjøtta, "Nonlinear effects in the farfield of a directive sound source", J. Acouct. Soc. Am. 78, 202-216 (1985).

5. T. S. Hart and M. F. Hamilton, "Nonlinear effects in focused sound beams",J. Acouct. Soc. Am. 84, 1488-1496 (1988).

6. A. C. Baker, K. Anastasiadis and V. F. Humphrey, "The nonlinear pressure field of a plane circular piston : Theory and experiment", in: J. Acouct. Soc. Am. 84 1483-1487 (1988).

7. A. C. Baker and V.F. Humphrey, "Nonlinear propagation of short ultrasonic pulses in focused field", in: the 12th Intemational Symposium on Nonlinear Acoustics Proceedings, edited by M. F. Hamilton and D. T. Blackstock (Austin, Aug. 90),185-190.

8. T. H. Neighbors and L. Bjørn $\varnothing$, "Focused Finite-Amplitude Ultrasonic Pulses in Liquids", in: the 12 th International Symposium on Nonlinear Acoustics Proceedings, edited by M. F. Hamilton and D. T. Blackstock (Austin, August 1990), pp 209-214.

9. S. El Nachef, J.Y. Chapelon and D. Cathignol, "Nonlinear pressure fields of focused transducers : theory and experiment", in: Ultrasonics International 91 conference Proceedings (to be published).

10. J. C. Lockwood and J. G. Willette, "High-speed method for computing the exact solution for the pressure variations in the nearfield of a baffled piston", J. Acouct. Soc. Am. 53 735-741 (1973). 
Froquence peincipale : $1 \mathrm{MHz}$

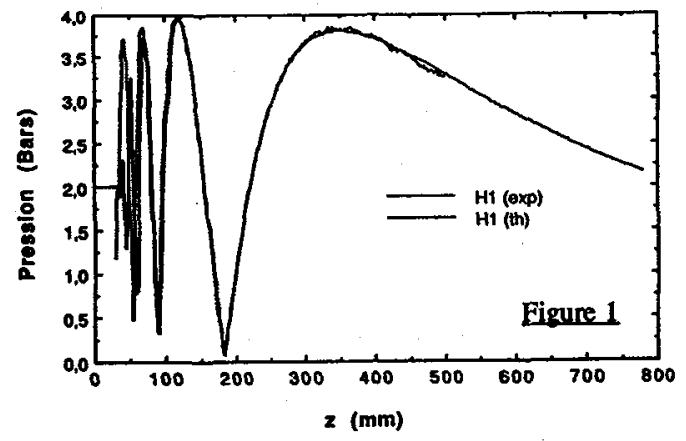

Trolslëme harmonique : $3 \mathbf{~ M H z}$

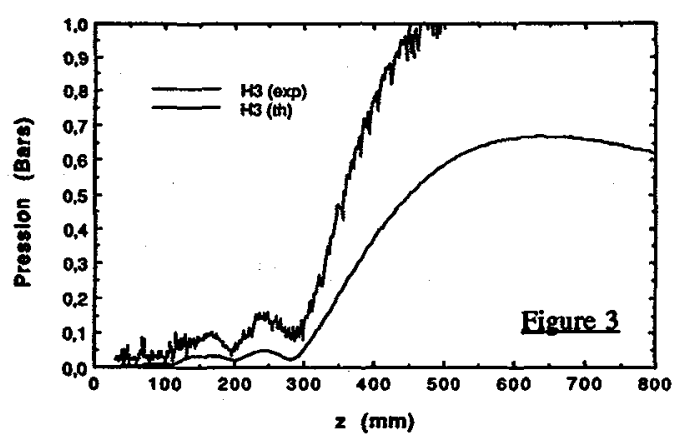

Evolution du premier harmonique sur l'axe

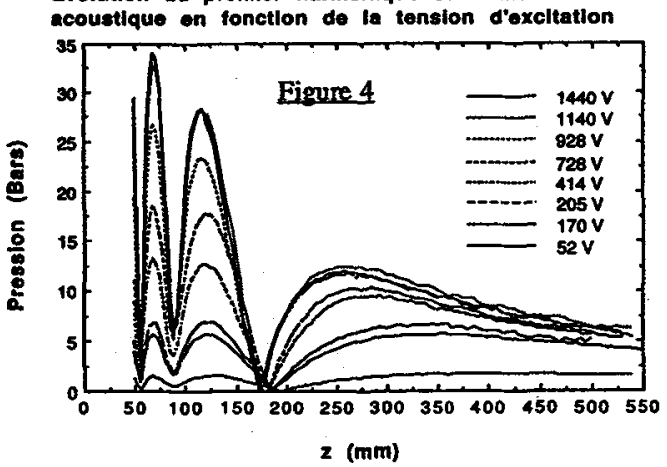

Evolution du trolsieme harmonlque sur l'axe acoustique on fonction de la tension diexcitation

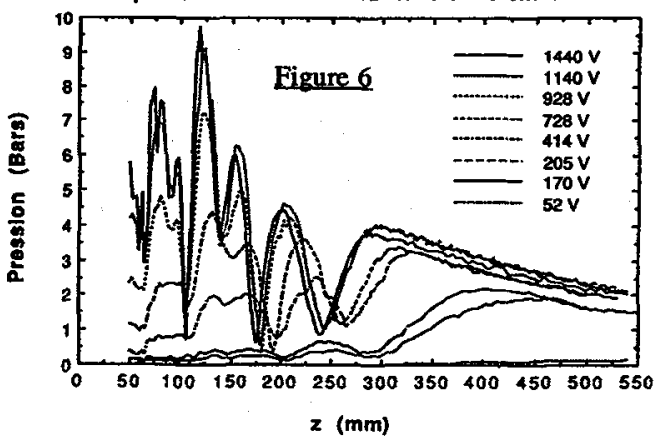

Deuxieme harmonique : $2 \mathrm{mHz}$

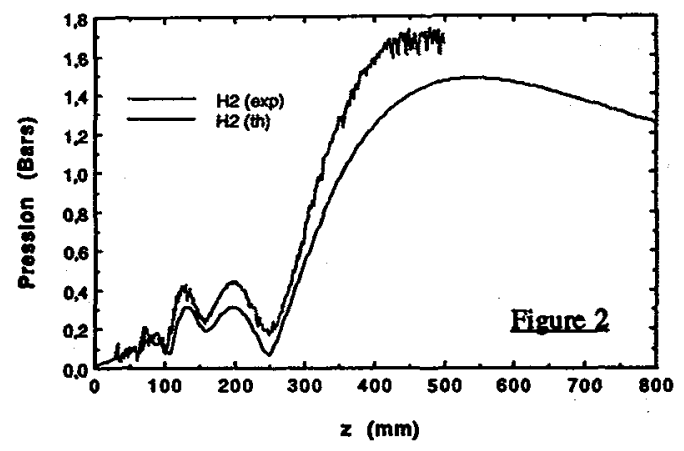

Comparaison des resultats experimentaux et calcules le long de l'axe acoustique :

Eigure 1: frequence principale de résonance du disque.

Eigure 2 : deuxième harmonique génére par effet non-linéaire.

Eiqure 3 : troisième harmonique génere par effet non-linéaire.

La pression a la source est de 2 Bars

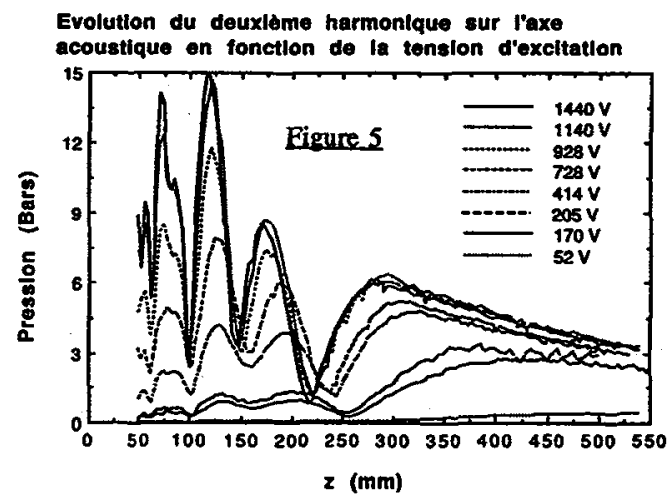

Evolution le long de l'axe acoustique et en fonction de la tension d'excitation des amplitudes des trois premiers harmoniques du champ ultrasonore mesure :

Figure 4 : fréquence principale de résonance (premier harmonique).

Fiqure 5 : deuxième harmonique généré par effet non-linéaire.

Eigure 6 : troisième harmonique généré par effet non-lin€aire. 